\title{
Prompt Charmonium Production at Belle
}

\author{
Stephen Schrenk* \\ 400 Geology/Physics \\ Cincinnati, OH 45221-0011, USA \\ E-mail: 'schrenk@phsyics.uc.edu'
}

Abstract: We have studied the prompt production of $J / \psi, \psi(2 S), \chi_{c 1}$, and $\chi_{c 2}$ at and near the $\Upsilon(4 S)$ resonance in a data set corresponding to $32.4 \mathrm{fb}^{-1}$ with the Belle detector at the KEKB $e^{+} e^{-}$collider. We find $\sigma\left(e^{+} e^{-} \rightarrow J / \psi X\right)=1.47 \pm 0.10 \pm 0.13$ pb. We set an upper limit for the production of $J / \psi$ directly from the $\Upsilon(4 S)$ resonance of $\mathcal{B}\left(\Upsilon(4 S) \rightarrow<1.9 \times 10^{-4}\right)$ at the $98 \%$ confidence limit. The cross-sections for prompt $\psi(2 S), \chi_{c 1}$, and $\chi_{c 2}$ are measured. The $J / \psi$ momentum spectrum, production angle distribution, and polarization are also studied.

The production processes for prompt charmonium are not well understood. Issues such as short distance vs. long distance effects and color-singlet vs. color octet production have been discussed in the literature [i]. Non-relativistic QCD (NRQCD) has provided

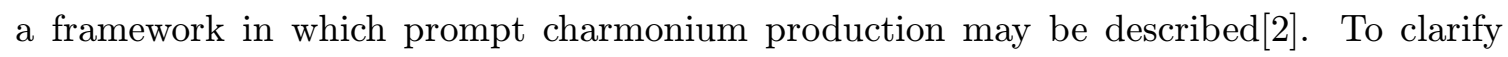
the picture additional studies of charmonium production are needed. The large data set available at and near the $\Upsilon(4 S)$ resonance allows us to make a variety of measurements that can test NRQCD.

The data set consists of $29.1 \mathrm{fb}^{-1}$ recorded at the $\Upsilon(4 S)$ resonance and an additional 3.0 $\mathrm{fb}^{-1}$ recorded $60 \mathrm{MeV}$ below the resonance (the continuum data set). It was collected with the Belle detector at the KEKB asymmetric energy $e^{+} e^{-}$storage ring $\left(E_{e^{+}}=3.5 \mathrm{GeV}, E+\right.$ $\left.e^{-}=8.0 \mathrm{GeV}\right)$.

Events are reconstructed with the Belle detector which is described in detail elsewhere[3]. The detector consists, from the center outward, of a three layer silicon vertex detector (SVD), a 50 layer central drift chamber (CDC), a mosaic of aerogel Cherenkov detectors for hadron identification (ACC), time-of-flight system (TOF) and a $\mathrm{CsI}(\mathrm{Tl}$ ) electromagnetic calorimeter (CSI). These are all inside a 1.5 Tesla superconducting coil. The iron flux return is instrumented to detect $K_{L}$ showers and muons (KLM).

Electron identification is based on a combination of CDC $d E / d x$ information, ACC response and the associated CSI shower energy and shape. Muon identification is based

${ }^{*}$ Speaker. 
on the depth a particle penetrates the KLM and the scatter of KLM hits along the track. Kaon and pions are identified by combining CDC $d E / d x$, TOF measurements and ACC response. Photons are identified by ECL showers not matched to a charged track and with a minimum energy of $20 \mathrm{MeV}$.

Events are first selected with a set of criteria designed to eliminate beam-gas, QED, and $\tau \tau$ backgrounds. These include requiring at least three "good" charged tracks that come from the interaction point, more than one "good" calorimeter cluster (energy greater than $100 \mathrm{MeV}$ ), a balanced momentum in the center of mass, and a value of $R_{2} \equiv H_{2} / H_{0} \leq 0.5$,

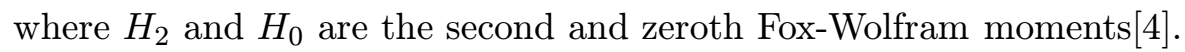

We reconstruct $J / \psi$ mesons through their decays to two leptons. Both leptons must be identified. Electrons suffer from both final state radiation and bremsstrahlung. In order to reduce the effect of these energy losses we include every photon detected with 0.05 radians of the initial electron direction in the invariant mass calculations. The two lepton tracks are required to have a common vertex with that is within $500 \mu \mathrm{m}$ of the interaction point in $r-\phi$ ( $z$ is along the $e^{-}$beam direction). In both cases the invariant mass is required to be within -93 and $+33 \mathrm{MeV} / \mathrm{c}^{2}$ of the known $J / \psi$ mass.

We reconstruct $\psi(2 S)$ through its decay to $J / \psi \pi^{+} \pi^{-}$. The $\pi^{+} \pi^{-}$vertex is required to be consistent with the $J / \psi$ vertex. We reconstruct $\chi_{c 1}$ and $\chi_{c 2}$ through their decays to $J / \psi \gamma$. Photons candidates are vetoed if when combined with another photon candidate they form a $\pi^{0}$ candidate.

The largest background in the $\Upsilon(4 S)$ data set is charmonium production from $B$ meson decay. To remove this background we require that the charmonium momentum in the center of mass $\left(p^{*}\right)$ be greater than $2.0 \mathrm{GeV} / \mathrm{c}$. This is above the kinematic limit for charmonium production coming from $B$ mesons. This requirement is not applied for the data sample taken below the $\Upsilon(4 S)$ resonance.

Another significant background comes from initial state radiation with a hard photon [5]. Backgrounds from higher order QED processes $\left(e^{+} e^{-} \rightarrow J / \psi \gamma^{*}, J / \psi l^{+}, l^{-}\right)$are also large [6] with 3 charged tracks. The $J / \psi$ mass peak has a signal to background ratio $(S / B)$ of about 0.1 . This is too poor to include in our final event sample. For events with 4 charged tracks, $S / B$ is acceptable but $85 \%$ of the $J / \psi$ mesons are due to QED processes (typically $\left.e^{+} e^{-} \rightarrow \psi(2 S) \gamma \rightarrow J / \psi \pi^{+} \pi^{-} \gamma\right)$, where the photon may or may not be detected). Based on these studies we require that there be at least 5 charged tracks in an event for our final data sample. Additional QED backgrounds in the final sample are removed by rejecting events with a $\psi(2 S) \rightarrow J / \psi \pi^{+} \pi^{-}$candidate accompanied by either a photon with center of mass energy greater than $3.5 \mathrm{GeV}$ or electron tracks consistent with a photon conversion.

Mass distributions for the final event sample are shown in Fig. In]. In the $\Upsilon(4 S)$ data sample there are $143 \pm 19 \mathrm{~J} / \psi \rightarrow e^{+} e^{-}$and $\mu^{+} \mu^{-}$events while in the data taken 60 $\mathrm{MeV}$ below the $\Upsilon(4 S)$ resonance there are $226 \pm 23$ events. The detection efficiency for $J / \psi \rightarrow e^{+} e^{-}$is $38 \%$ while for $\mu^{+} \mu^{-}$it is $48 \%$.

In Fig. $12 \mathrm{a}$ a , the mass difference distribution, $M_{l^{+} l^{-} \pi^{+} \pi^{-}}-M_{l^{+} l^{-}}$, for the $\Upsilon(4 S)$ data set is plotted. A $\psi(2 S)$ signal of $143 \pm 19$ events can be seen. No statistically significant signal $(10 \pm 5)$ is seen in the continuum data set. In Fig. ${ }_{2 i}^{2} \mathrm{~b}$, the mass difference distribution, 


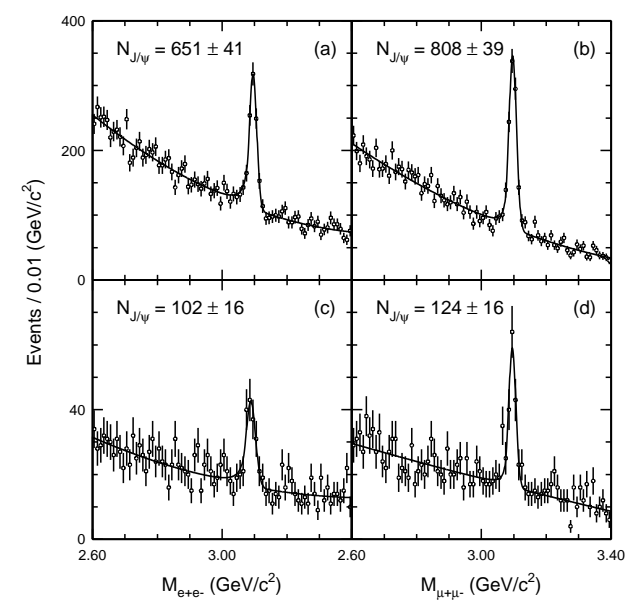

Figure 1: Mass distributions for $J / \psi \rightarrow$ $e^{+} e^{-}(\mathrm{a}, \mathrm{c})$, and $\mu^{+} \mu^{-}(\mathrm{b}, \mathrm{d})$, for $\Upsilon(4 S)$ (a, b) and continuum (c, d) data.
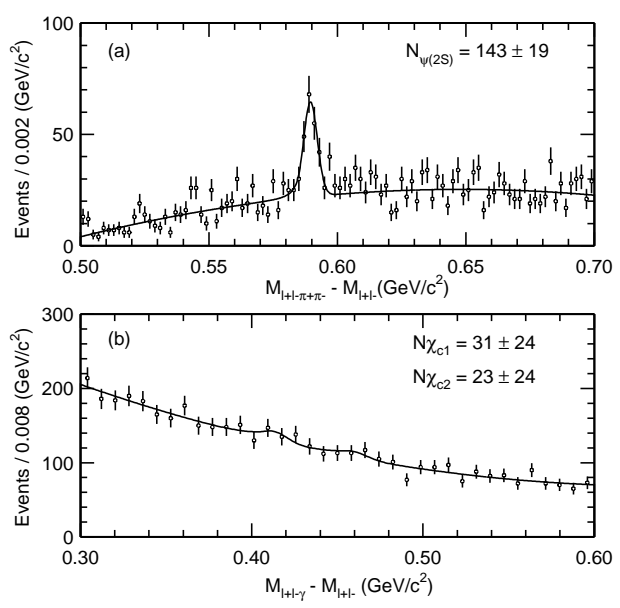

Figure 2: Mass difference distributions for $\Upsilon(4 S)$ data for (a) $\psi(2 S) \quad\left(M_{l^{+} l^{-} \pi^{+} \pi^{-}}-\right.$ $\left.M_{l^{+} l^{-}}\right)$, (b) $\chi_{c 1}$ and $\chi_{c 2}\left(M_{l^{+} l^{-} \gamma}-M_{l^{+} l^{-}}\right)$.

$M_{l^{+} l^{-} \gamma}-M_{l^{+} l^{-}}$, for the $\Upsilon(4 S)$ data set is plotted. No significant $\chi_{c 1}$ or $\chi_{c 2}$ signal can be seen.

We compare $J / \psi$ production from $p^{*}>2.0 \mathrm{GeV} / c$ in the $\Upsilon(4 S)$ and continuum data sets by fitting the invariant mass distributions in $300 \mathrm{MeV} / c$ wide bins of $p^{*}$ for $J / \psi$ yield. The resulting yield distributions agree quite well between the two data sets when normalized to the data set sizes. We then extract an upper limit on non- $B$-meson $J / \psi$ production from the $\Upsilon(4 S)$. The normalized yields for the $\Upsilon(4 S)$ data set and for the continuum data set are $1459 \pm 57$ and $1498 \pm 145$ respectively. Following the method of Feldman-Cousins [i] we set an upper limit at the $95 \%$ confidence limit of $\mathcal{B}(\Upsilon(4 S) \rightarrow J / \psi X)<1.9 \times 10^{-4}$. This is lower than the result of Ref. [i⿱⿱亠䒑日心]. The ratio of $\sigma(\psi(2 S) X) / \sigma\left(J / \psi_{\text {direct }}\right)$ is measured to be $0.93 \pm 0.11_{-0.15}^{+0.13}$.

From this point on we will assume that all charmonium with $p^{*}>2.0 \mathrm{GeV} / c$ in the $\Upsilon(4 S)$ data set are due to continuum production, allowing us to combine the two data sets. $\mathrm{GeV} / c$. In order to find production cross sections and angular distributions we need acceptance corrections. These are determined from a Monte Carlo simulation of $e^{+} e^{-} \rightarrow J / \psi(\psi(2 S)) q \bar{q}$ where the composition of $q \bar{q}$ is set to that observe at $\sqrt{s} \approx 10.6$ $\mathrm{GeV}$. To reduce model dependence, the acceptance is determined in 15 bins of $p^{*}$ and $\cos \theta^{*}$ (the $J / \psi$ production angle in the center of mass system). The momentum region $p^{*}>2.0$ $\mathrm{GeV} / c$ is divided into 3 regions while $\cos \theta^{*}$ is divided into 5 regions. Each bin is fitted and corrected separately for $J / \psi$ and for $\psi(2 S)$ yield. For $\chi_{c} 1$ and $\chi_{c 2}$ no binning was done.

The resulting cross-sections are shown in Table contribution from $\psi(2 S)$ to $J / \psi$. This is measured to be $0.33 \pm 0.44_{-0.05}^{+0.05} \mathrm{pb}$. It is subtracted from the measured $J / \psi$ cross section to obtain the direct $J / \psi$ cross section. To obtain the cross section for $J / \psi$ production for the entire momentum range, the off-resonance data is added. In the off-resonance data we see no significant signal for continuum $\psi(2 S), \chi_{c 1}$, or $\chi_{c 2}$ production thus we estimate cross sections for $p^{*}>2.0 \mathrm{GeV} / c$ only.

The systematic errors in the cross-section measurements include (where applicable): 


\begin{tabular}{lcc}
\hline \hline$\sigma$ & $0<p^{*}<p_{\text {max }}^{*}$ & $\begin{array}{c}2.0<p^{*}<p_{\max }^{*} \\
(\mathrm{GeV} / c)\end{array}$ \\
$(\mathrm{pb})$ & $(\mathrm{GeV} / c)$ & $1.05 \pm 0.04 \pm 0.09$ \\
\hline$\sigma\left(e^{+} e^{-} \rightarrow J / \psi X\right.$ & $1.47 \pm 0.10 \pm 0.13$ & $0.72 \pm 0.08_{-0.17}^{+0.13}$ \\
$\sigma\left(e^{+} e^{-} \rightarrow J / \psi_{\text {direct }} X\right.$ & - & $0.67 \pm 0.09_{-0.11}^{+0.09}$ \\
$\sigma\left(e^{+} e^{-} \rightarrow \psi(2 S) X\right.$ & - & $<0.35$ \\
$\sigma\left(e^{+} e^{-} \rightarrow \chi_{c 1} X\right.$ & - & $<0.66$ \\
$\sigma\left(e^{+} e^{-} \rightarrow \chi_{c 2} X\right.$ & - & $<$ \\
\hline \hline
\end{tabular}

Table 1: The measured charmonium cross-sections. The upper limits for $\chi_{c 1}$ and $\chi_{c 2}$ are at the 90\% Confidence Limits.

efficiency uncertainty $( \pm 5 \%)$, efficiency of multiplicity cut $(+4 \%)$, tracking $( \pm 4 \%)$, luminosity measurement $( \pm 1.3 \%)$, possible feed-down from $\chi_{c 1}$ and $\chi_{c 2}(-10 \%)$, QED contamination $(-4 \%), \psi(2 S)$ feed-down subtraction $( \pm 2 \%)$, and daughter branching fractions. Source of systematic error that were found to be negligible include: contamination from $\Upsilon(1 S, 2 S, 3 S) \gamma$, and two photon processes. We also check $J / \psi$ cross sections from $J / \psi \rightarrow e^{+} e^{-}$and $\mu^{+} \mu^{-}$separately. The ratio between the two is $1.00 \pm 0.07 \pm 0.04$.

Our $J / \psi$ cross sections are smaller than those reported by BaBar and the $\psi(2 S)$ results are first measurements.

The efficiency corrected momentum distributions for $J / \psi$ and $\psi(2 S)$ are shown in Fig. $\underline{\beta}_{3} \mathrm{a}$ and Fig. ${ }_{13}^{1} \bar{i} \mathrm{i}$ respectively. For $J / \psi$, the feed-down from $\psi(2 S)$ is also shown. The $J / \psi$ distribution ends about $300 \mathrm{MeV} / c$ below the kinematic limit $(4.84 \mathrm{GeV} / c)$. The distribution is softer than NRQCD predicts for color-singlet $J / \psi g g$ production [9i] [1] [1] The end-point cross section does not rise as some NRQCD color-octet $J / \psi g$ production

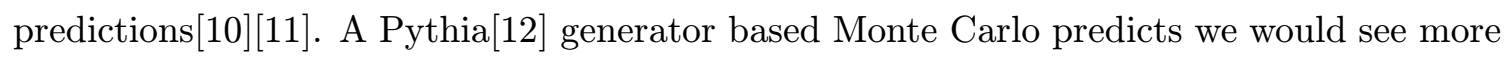
than 300 events in the last two bins of Fig. $\overline{3}$ a assuming a 1 pb cross section for this coloroctet production mechanism. Clearly this is not the case. The $\psi(2 S)$ distribution does extends to the kinematic end point $(4.65 \mathrm{GeV} / c)$.

Angular distributions are shown in Fig. 商 for both $\theta^{*}$ and $\theta_{H}$ (the angle between the positive lepton daughter momentum vector in the $J / \psi$ rest frame and the $J / \psi$ momentum vector in the center of mass system). The yields are corrected for efficiency but not for feed-down from $\psi(2 S)$. The distributions are fitted with the parameterizations $1+A \cos ^{2} \theta^{*}$ and $1+\alpha \cos ^{2} \theta_{H}$. Results of the fits are shown in Table ${ }_{-i}^{1}$ No statistically significant $p^{*}$ dependence is seen for either parameter.

\begin{tabular}{ccccc}
\hline \hline$p_{J / \psi}^{*}(\mathrm{GeV} / c)$ & $A$ & $\chi^{2} /$ d.o.f & $\alpha$ & $\chi^{2} /$ d.o.f. \\
\hline $2.0-2.6$ & $0.3_{-0.4}^{+0.5}$ & $1.5 / 4$ & $-0.4 \pm 0.2$ & $7.8 / 4$ \\
$2.6-3.4$ & $1.1_{-0.4}^{+0.4}$ & $5.0 / 4$ & $-0.4 \pm 0.1$ & $1.3 / 4$ \\
$3.4-4.9$ & $1.1_{-0.3}^{+0.4}$ & $4.5 / 4$ & $-0.2 \pm 0.2$ & $7.1 / 4$ \\
\hline $2.0-3.4$ & $0.7 \pm 0.3$ & $1.2 / 4$ & $-0.5 \pm 0.1$ & $7.8 / 4$ \\
$2.0-4.9$ & $1.9 \pm 0.2$ & $3.0 / 4$ & $-0.4 \pm 0.1$ & $13.4 / 4$ \\
\hline \hline
\end{tabular}

Table 2: Results of the fits to angular distributions. 


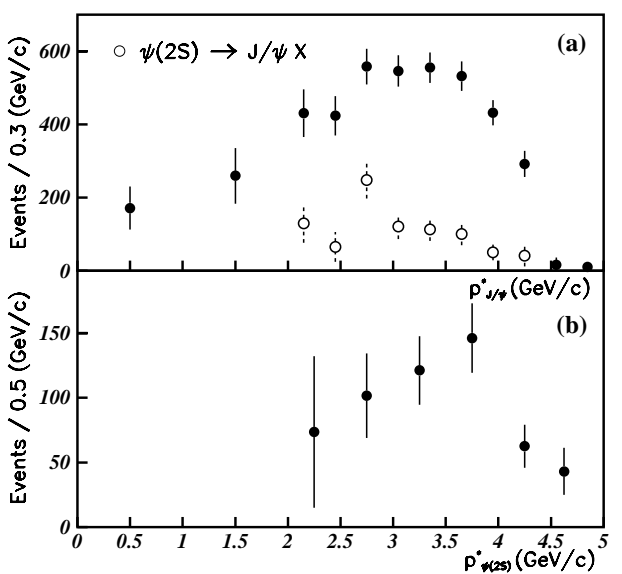

Figure 3: Center of mass momentum distributions for prompt charmonia (efficiency corrected): (a) $J / \psi$ (filled points), $J / \psi$ from $\psi(2 S) \rightarrow J / \psi X$ (open points), (b) $\psi(2 S)$.
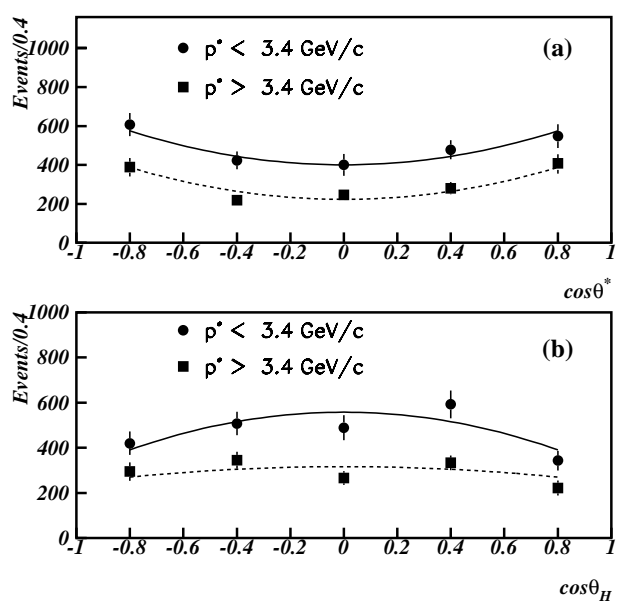

Figure 4: The $\cos \theta^{*}$ (a) and $\cos \theta_{H}$ distributions for low and high $p^{*}$. The curves represent fits. See the text for details.

The color-singlet $J / \psi c \bar{c}$ process should result in a large value for $A[\overline{1} \overline{1} \overline{0}]$. The expected

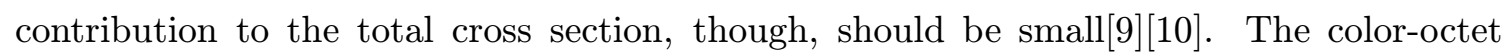
process $J / \psi g$ should produce a value for $A$ near 1 at the endpoint [i to be less that -0.4 for the color-singlet process $J / \psi g g\left[\bar{p}_{\bar{p}}\right]$.

In summary, we have measured the cross sections from $J / \psi$ and $\psi(2 S)$ production at energies near the $\Upsilon(4 S)$ mass. We set an upper limit for the process $\Upsilon(4 S) \rightarrow J / \psi X$. These results are in a paper recently accepted by Phys. Rev. Lett [ī $\underline{1}_{\overline{1}} \overline{1}$.

\section{References}

[1] M. Kramer, hep-ph/0106120, to be published in Prog. Part. Nucl. Phys.

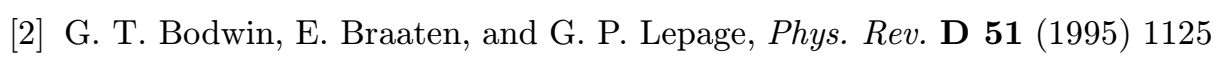

[3] K. Abe et al. (Belle Collaboration), KEK Report 2000-4, to be published in Nucl. Instrum. Methods Phys. Res.

[4] G. C. Fox and S. Wolfram, Phys. Rev. Lett. 41 (1978) 1581!

[5] M. Benayoun, S. I. Eidelman, and Z. K. Silagadze, Mod. Phys. Lett. A $1 \overline{4}(19 \overline{9} 9 \overline{2}) \overline{2} 6 \overline{5}$.

[6] C.-H. Chang, C.-F. Qiao, and J.-X. Wang, 'Phys. Rev. D 57 (1998) 3873.

[7] G. J. Feldman and R. D. Cousins, 'P $\mathrm{P} h y s$. Rev. D $5 \overline{7}(19 \overline{9} 8)$ 3873.'.

[8] B. Aubert (BaBar Collaboration), i'

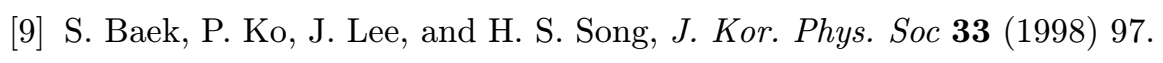

[10] P. Cho and A. K. Leibovich, 'Phys. Rev. D 54 (1996) 6690.

[11] E. Braaten and Yu-Qi Chen, 'Phys. Rev. Lett. 54

[12] T. Sjöstrand, Pythia 5.7/Jetset 7.4, CERN-TH.7112/93 (1993); T. Sjöstrand, 'Comp. Phys.! (- Commun. $\mathbf{8 2}(199 \overline{9}) \overline{4}$

[13] K. Abe et al. (Belle Collaboration), to be published in Phys. Rev. Lett. (2001). 